\title{
Enhancement of Protein Sensitivity for MALDI Imaging Mass Spectrometry After Chemical Treatment of Tissue Sections
}

\author{
Erin H. Seeley, Stacey R. Oppenheimer, ${ }^{*}$ Deming Mi, Pierre Chaurand, \\ and Richard M. Caprioli \\ Mass Spectrometry Research Center and Department of Biochemistry, Vanderbilt University School of \\ Medicine, Nashville, Tennessee, USA
}

MALDI imaging mass spectrometry (IMS) has become a valuable tool for the investigation of the content and distribution of molecular species in tissue specimens. Numerous methodological improvements have been made to optimize tissue section preparation and matrix deposition protocols, as well as MS data acquisition and processing. In particular for proteomic analyses, washing the tissue sections before matrix deposition has proven useful to improve spectral qualities by increasing ion yields and the number of signals observed. We systematically explore here the effects of several solvent combinations for washing tissue sections. To minimize experimental variability, all of the measurements were performed on serial sections cut from a single mouse liver tissue block. Several other key steps of the process such as matrix deposition and MS data acquisition and processing have also been automated or standardized. To assess efficacy, after each washing procedure the total ion current and number of peaks were counted from the resulting protein profiles. These results were correlated to on-tissue measurements obtained for lipids. Using similar approaches, several selected washing procedures were also tested for their ability to extend the lifetime as well as revive previously cut tissue sections. The effects of these washes on automated matrix deposition and crystallization behavior as well as their ability to preserve tissue histology were also studied. Finally, in a full-scale IMS study, these washing procedures were tested on a human renal cell carcinoma biopsy. (J Am Soc Mass Spectrom 2008, 19, 1069-1077) ( 2008 American Society for Mass Spectrometry

I maging mass spectrometry (IMS) is becoming a key technology for the investigation of the molecular content of thin tissue sections in direct spatial correlation with the underlying histology [1-4]. An analysis by matrix-assisted laser desorption/ionization mass spectrometry (MALDI-MS) performed directly on a tissue section allows detection of a wide range of endogenous molecular species such as lipids $[5,6]$, peptides [7-10], and proteins [1, 3, 11] as well as administered pharmaceuticals [12-15]. Typically, several hundred distinct protein mass signals are observed in a single analysis in a mass-to-charge $(\mathrm{m} / \mathrm{z})$ range up to 70,000 , although the technology is capable of measurements exceeding 200,000 [16]. In IMS, the homogeneous deposition of the MALDI matrix followed by a systematic acquisition of data allows reconstruction of hundreds of distinct ion signal intensity maps, or images,

Address reprint requests to Dr. Richard M. Caprioli, Vanderbilt University School of Medicine, Mass Spectrometry Research Center, Departments of Chemistry and Biochemistry, 9160 MRB III, Nashville, TN 37232-8575. E-mail: r.caprioli@vanderbilt.edu

* Present address: Pfizer Global Research and Development, Groton, CT 06340. for virtually all of the different molecular species observed [1,3]. IMS has been used to study a wide variety of animal and plant systems [17]. Particular emphasis has been given to the study of disease tissues from both the biological and the clinical perspectives [18-20]. Of particular interest is the fact that the expression of subsets of protein signals could be correlated to the progression of disease as well as patient outcome.

Over the last decade, numerous methodological and technical developments have emerged to improve upon sample preparation for protein analyses. Efforts in sample preparation have the goal of increasing the quality and reproducibility of the mass spectrometric data. Schwartz et al. [21] first defined a set of practical guidelines for the proper handling of tissue sections including tissue storage, sectioning, and mounting for peptide and protein analyses. In the same article, other parameters such as the choice and concentration of matrix, solvent composition, matrix deposition methodologies, and instrumental parameters for optimal mass spectrometric data acquisition are discussed [21]. The influence of the tissue thickness (between 2 and $40 \mu \mathrm{m}$ ) has also been studied [22]. The authors concluded that 
the overall peak intensities and the total number of observed peaks drastically increased as the sections became thinner. Several MALDI-compatible tissue staining protocols combined with the use of conductive indium-tin oxide-coated glass slides for tissue mounting have been developed to directly link the observed histological features with the recovered mass spectrometric data [23]. Several tissue-rinsing procedures before matrix application have also been examined [3, 24].

Matrix deposition strategies and procedures aimed toward proteomic analyses have dramatically evolved. The first tissue imaging experiments were performed after manually spreading one large drop of matrix over the tissue section $[1,25]$. Although in some instances this procedure is successful, this matrix deposition approach often leads to a significant degree of analyte delocalization. Matrix deposition by pneumatic spray coating is largely used for homogeneous deposition [11, 21]. In some cases this approach has the disadvantage of significantly degrading the quality of the mass spectrometric data and to some extent delocalizing the analytes [16]. More recently, the development of technologies for automated dispensing of picoliter size droplets of matrix on the sections into independent spots of approximately 100-200 $\mu \mathrm{m}$ diameter have circumvented the signal quality and delocalization aspects but limit the imaging resolution to the center-to-center distance between adjacent spots [3, 26, 27]. Because of the high spotting accuracy of the automated dispensing approaches, a histology-directed matrix deposition strategy to target precise areas of the tissue sections has been developed [4]. Further, these spotters have also been used to first print enzymatic proteases before IMS [28, 29]. This approach allows for direct on-tissue identification of proteins in spatially discrete regions using enzymatic digestion followed by IMS and MALDI tandem mass spectrometric (MS/MS) analyses of the resulting peptides. This strategy has been successfully used to analyze the proteomic content of sections from formalin-fixed paraffin-embedded tissue specimens [30].

Finally, MS instrumentation for both the acquisition of IMS data and post-acquisition data processing approaches has significantly progressed. Historically, IMS data were first collected on MALDI time-of-flight (TOF) mass spectrometers equipped with compact nitrogen lasers $(337 \mathrm{~nm})$. Additionally, an iris can be installed across the laser-beam pathway to control the irradiated tissue surface [1]. Homebuilt MALDI-TOF MS systems with laser-beam dimensions on target of $10 \mu \mathrm{m}$ or smaller have since been developed [31, 32]. Another homebuilt position-sensitive mass spectrometer has been described capable of generating images with a lateral resolution of better than $5 \mu \mathrm{m}$ [33]. Early commercially available MALDI-TOF systems allowed laser repetition rates of a few Hertz. Current systems with enhanced electronics and improved acquisition systems are now equipped with solid-state Nd:YAG lasers (355 $\mathrm{nm})$, functioning at repetition rates as high as 200 to 300 $\mathrm{Hz}[25,34]$. IMS has also been successfully performed on other types of commercially available MS instruments, such as QqTOF, ion traps, and more recently Fourier transform ion cyclotron resonance (FT-ICR) systems [7, 35, 36]. Whereas early software programs for IMS data acquisition and visualization were written "in-house" [31,37], today almost every commercially available MALDI-based mass spectrometer is "imaging capable" and can be equipped with software for automated data acquisition, image reconstruction, and visualization. IMS data preprocessing (background subtraction and noise estimation, peak alignments, normalization) and postprocessing (statistical analyses) have also been developed [38-41].

In this article, we explore tissue section rinsing protocols to improve IMS protein signal sensitivity. The action of washing or rinsing the sections before matrix application and MALDI IMS analyses is performed to remove contaminants such as tissue-embedding media as well as endogenous molecular species, such as lipids or biological salts, that may affect protein desorption/ ionization efficiency. The use of organic solvents to extract lipids from blood and tissues has been developed in the early to mid twentieth century [42]. In particular Folch and colleagues, and Bligh and Dyer have respectively developed and optimized mixtures of chloroform-methanol for the rapid extraction of lipids from animal tissues [43-45]. Lin et al. [46] tested a mixture of ethyl acetate and ethyl alcohol as an alternative to the Folch reagent for extracting animal lipids. Numerous other solvents and mixtures of solvents for lipid extractions have also been reported [47, 48]. The lipid removal potency of noncrosslinking chemical fixatives applied to tissue sections has also been reported [49]. When using ethanol, it was estimated that roughly $75 \%$ of the lipid content was extracted from rat liver and brain sections. Some organic solvents such as alcohols, chloroform, or acetone or mixtures of these are also used to precipitate proteins from solutions [46, 50]. These same solvents are also classically used in histotechnologies to dehydrate, fix, and generally preserve tissues [51]. For example, ethanol and xylene are routinely used to dehydrate sections during several staining procedures or before laser capture microdissection (LCM) of individual cells [52]. When thin tissue sections are subjected to these solvents before immunohistochemistry, LCM, or IMS, it is presumed that most proteins will precipitate in situ and stay localized within the cellular substructures.

We have observed early on that submerging sections in ethanol-based saturated matrix solutions to generate small seeding crystals did not negatively affect the IMS signal quality [21]. Similarly, no significant signal quality deterioration was observed during the development of the MALDI-compatible staining procedures [23]. We have recently established an ethanolbased tissue-rinsing procedure before the IMS analyses of proteins, which not only gives cleaner IMS data but also seems to better preserve the tissue sections over time [3]. Others have also studied several tissue-rinsing 
procedures. Lemaire et al. [24] studied the efficacy of chloroform, hexane, toluene, acetone, and xylene to remove lipids from the sections. In conjunction with lipid removal, they observed an increase in overall peptide and protein detection in the $\mathrm{m} / \mathrm{z}$ range from 5000 to 30,000 as well as increased signal reproducibility. Using a very systematic analytical approach including automated matrix dispensing technology in combination with automated MS data acquisition and processing, we examine the effect of numerous solvent systems on IMS analyses of fresh frozen mammalian tissue sections. Through the use of sections from a single mouse liver tissue specimen, parameters such as the number of peaks and overall protein $(\mathrm{m} / \mathrm{z}$ from 2000 to 25,000$)$ and lipid ( $m / z$ range from 500 to 2000$)$ total ion currents (TICs) are compared across solvent systems. For some of the solvent systems investigated, a study on the stability and recoverability of rinsed sections as a function of time is also examined. Finally, using a human renal cell carcinoma biopsy containing different histologies, IMS measurements were performed to assess individual signal variability as well as eventual delocalization behaviors.

\section{Experimental}

Ethanol, methanol, acetonitrile, acetone, acetic acid, isopropanol, and hexane were purchased from Fisher Scientific (Suwanee, GA, USA). Trifluoroacetic acid and xylene were purchased from Acros (Morris Plains, NJ, USA). Chloroform, tert-butyl methyl ester, sinapinic acid, and 2,6-dihydroxyacetophenone were purchased from Sigma-Aldrich (Milwaukee, WI, USA). Toluene was purchased from JT Baker (Phillipsburg, NJ, USA).

A control mouse liver was serially sectioned at 12 $\mu \mathrm{m}$ thickness on a Leica CM1900 cryostat (Leica Microsystems $\mathrm{GmbH}$, Wetzlar, Germany) and thaw-mounted onto gold-coated MALDI targets. For most analyses one section was collected per plate. Plates were placed in a desiccator to dry for $20 \mathrm{~min}$ before washing. The sections were washed by submerging the plates in 50 $\mathrm{mL}$ of solvent placed in a glass petri dish and gently swirling. Tissue washes were for $30 \mathrm{~s}$ per solution as follows:

1. $2 \times 100 \%$ acetone (acetone)

2. $70 \%$ ethanol followed by $95 \%$ ethanol (ethanol)

3. $70 \%$ isopropanol followed by $95 \%$ isopropanol (isopropanol)

4. $70 \%$ methanol followed by $95 \%$ methanol (methanol)

5. $70 \%$ ethanol followed by $90 \%$ ethanol $/ 9 \%$ acetic acid (acetic acid)

6. $2 \times 100 \%$ chloroform (chloroform)

7. $2 \times 100 \%$ toluene (toluene)

8. $2 \times 100 \%$ hexane (hexane)

9. $2 \times 100 \%$ xylene (xylene)

10. $2 \times 100 \%$ water (water)

11. $2 \times 100 \%$ tert-butyl methyl ether $(t$-BME)

12. No wash (control)
After washing, plates were tilted to maximize solvent removal and were returned to the desiccator to dry before matrix deposition.

Sinapinic acid [25 mg/mL in 50/0.1 (vol/vol) acetonitrile/trifluoroacetic acid] was used as the matrix for protein analysis, whereas 2,6-dihydroxyacetophenone [DHA, $10 \mathrm{mg} / \mathrm{mL} \mathrm{60/40} \mathrm{(vol/vol)} \mathrm{ethanol/water]} \mathrm{was}$ used as the matrix for phospholipid analysis. Discrete matrix spots printed in $5 \times 5$ arrays ( 25 spots per tissue section, $\sim 250 \mu \mathrm{m}$ diameter), were deposited using an acoustic robotic microspotter (Labcyte, Sunnyvale, CA, USA) [26]. Care was taken to deposit matrix to approximately the same location on each section while avoiding obvious blood vessels.

A time-course experiment consisting of two parts was carried out on some of the washes from the initial experiment. In the first, five plates were washed the same day they were sectioned with ethanol, isopropanol, water, or acetic acid. Washed tissue was returned to the desiccator to dry and stored until the day of analysis. Tissue was spotted and analyzed on Days 1, 2, 4, 7, and 14 after sectioning. In the second part, the plates were not immediately washed, but left in the desiccator and "revived" by washing on Days 2, 4, 7, and 14 after sectioning. This was accomplished by subjecting the plates to the same four washes listed earlier. Two controls were included in the time-course study. In the first, tissue was not washed, left in the desiccator, and spotted with matrix on the day of analysis. For the other control, sections were not washed, but spotted with matrix the day they were sectioned and then left in the desiccator until mass spectral analysis was conducted on a later date.

All of the MALDI mass spectrometric measurements were performed on a Bruker Autoflex II (Billerica, MA, USA) equipped with a SmartBeam laser (Nd:YAG, 355 $\mathrm{nm}$ ) and an electrostatic reflector. Protein mass spectra were acquired from each spot in an automated fashion in linear positive-ion mode. The nominal accelerating voltage was $20 \mathrm{kV}$ with an extraction voltage of 18.65 $\mathrm{kV}$, an Einzel lens voltage of $6.00 \mathrm{kV}$, and a delay time of 350 ns. Delayed extraction parameters were optimized for optimal resolution at $12 \mathrm{kDa}$. Lipid spectra were acquired in both positive- and negative-ion reflector modes. Positive-ion mode lipid spectra were acquired with an accelerating voltage of $19 \mathrm{kV}$, an extraction voltage of $17 \mathrm{kV}$, a delay time of $100 \mathrm{~ns}$, a lens voltage of $8 \mathrm{kV}$, and a reflector voltage of $20 \mathrm{kV}$. Negative-ion mode spectra were collected using an accelerating voltage of $-19 \mathrm{kV}$, an extraction voltage of $-17 \mathrm{kV}$, a delay time of $100 \mathrm{~ns}$, a lens voltage of -8.2 $\mathrm{kV}$, and a reflector voltage of $-20 \mathrm{kV}$. For both positiveand negative-ion mode lipid spectra, delayed extraction parameters were optimized for optimal resolution at $800 \mathrm{Da}$. All spectra were the sum of 500 individual laser shots collected in 50-shot increments within each matrix spot. The laser position was moved within the spot between each incremental step.

Spectra were preprocessed using software from Biodesix, Inc. (Steamboat Springs, CO, USA), which 
included baseline correction and alignment to common peaks and averages generated for each wash type. Evaluation of results included comparison of TIC, number of peaks, and intensity of background. Spectra were also visually examined for the presence or absence of signals.

Mass spectral images were acquired from sections of human clear cell renal cell carcinoma samples that had been subjected to each of the washes used in the time-course study. All sections were mounted and simultaneously imaged on a single MALDI target. Sinapinic acid was applied in a $55 \times 12$ grid across each section with a lateral resolution of $300 \mu \mathrm{m}$ using a Portrait 630 Multireagent Spotter (Labcyte). Discrete matrix spots were deposited in fly-by mode with 20 passes of three drops each. Protein spectra were acquired on a Bruker Autoflex II in linear mode geometry, as described earlier, using the same instrumental parameters. Each pixel was a composite of 200 laser shots acquired in 50-shot increments from four positions within a single matrix spot. Images were visualized using BioMap software (Novartis, Basel, Switzerland).

Photomicrographs of the tissue sections after matrix deposition were obtained at $\times 4$ and $\times 10$ magnification using an Olympus BX50 microscope (Olympus America Inc., Center Valley, PA, USA) equipped with a Micropublisher 3.3 RTV digital camera (Q Imaging, Surrey, BC, Canada). Hematoxylin and eosin (H\&E) section staining was performed according to traditional protocols. Magnified photomicrographs of the H\&E stained tissue sections were obtained after scanning the slides using a Mirax Desk microscope slide scanner (Zeiss, Thornwood, NY, USA) at a pixel resolution of $0.23 \mu \mathrm{m}$. Digital photomicrographs were exported using the Mirax Viewer software.

\section{Results and Discussion}

Sample preparation is of the utmost importance when carrying out direct tissue analysis by IMS. Care must be taken to ensure that the sample is handled properly from the time it is removed from the organism up through mass spectral analysis. Tissues should be snapfrozen immediately after resection to prevent proteolytic degradation and kept frozen at $-80{ }^{\circ} \mathrm{C}$. Sections should be thoroughly thawed when mounted onto a MALDI target because residual moisture can lead to freezer burn. Fixation or washing can greatly improve protein signal quality and prolong the viable life of the tissue. Matrix deposition is important in determining the subset of proteins extracted from the tissue and detected [3]. Careful selection of acquisition parameters on the mass spectrometer is necessary to optimize for the mass range in which the signals of interest fall.

The solvents used in this study were selected either based on their known efficacy for lipid solubilization and tissue preservation, or have been previously reported for washing tissue sections before analyses by mass spectrometry. A systematic study was undertaken in which 11 different tissue-washing approaches were examined for their influence on protein and lipid signal. From the results of this, four of these washes were used to assess their ability to preserve the tissue for protein analysis on a later date and for the possibility that tissue could be "revived" by washing several days after sectioning. Finally, protein images were carried out on serial sections of a human clear cell renal cell carcinoma to visually assess the degree of protein loss induced by the washing techniques and the extent of protein delocalization.

The first part of the study involved exploring 11 different tissue-washing approaches for their effect on protein and lipid signals. To minimize variability coming from the sample, all of the experiments were performed on serial sections originating from a single mouse liver lobe. Liver was used because of its relative homogeneity over a large volume. Sections of control mouse liver were thaw-mounted onto gold-coated MALDI targets, subjected to the 11 wash methods listed earlier and spotted with sinapinic acid for protein analysis. To monitor intrasample variability, each tissue section was spotted with a $5 \times 5$ matrix spot array and analyzed by mass spectrometry. After data collection, the 25 resulting spectra were baseline corrected, aligned, and peaks selected. The average traces from the processed spectra from each wash as well as the nonwashed control are displayed in Figure 1a. Although a fair majority of the signals observed are very similar for all of the washes (e.g., Figure 1b), there are some notable differences. For example, $m / z$ 9912, which was the base peak observed after most of the washes, is completely devoid from the water average spectrum (Figure 1d). Additionally, the signal at $m / z 13,777$ is readily apparent only in the tissue sections washed with water, chloroform, and acetic acid (Figure 1e). Figure 1c displays a pair of signals that show an interesting trend; two signals are observed at $m / z 7928$ and 7936, which appear to be specific to the type of wash used. The signal at $m / z 7928$ shows a strong preference for chloroform and acetic acid, whereas $\mathrm{m} / \mathrm{z} 7936$ shows a strong preference for methanol and isopropanol. The other washes tend to show one or the other of the signals with hexane showing equal distribution of both. The protein spectra were also evaluated for TIC, background, noise, and number of observed peaks. Plots showing TIC and number of peaks as a function of wash type are shown in Figure $2 a$ and b, respectively. The different washes were ordered with increasing protein TIC. The TIC was found lowest for acetone and highest for isopropanol. A simple linear fit was drawn to guide the eye. Interestingly, in a comparison of all washes the increase in the number of peaks observed was found proportional to the increase in TIC. This behavior was also found roughly true for background and noise (not shown). The total number of peaks tracked ranged from 85 for the acetone-washed tissues to 129 for the methanol-washed tissue.

The washed and control mouse liver sections were also spotted with $5 \times 5$ arrays of 2,6-dihydroxyacetophenone 


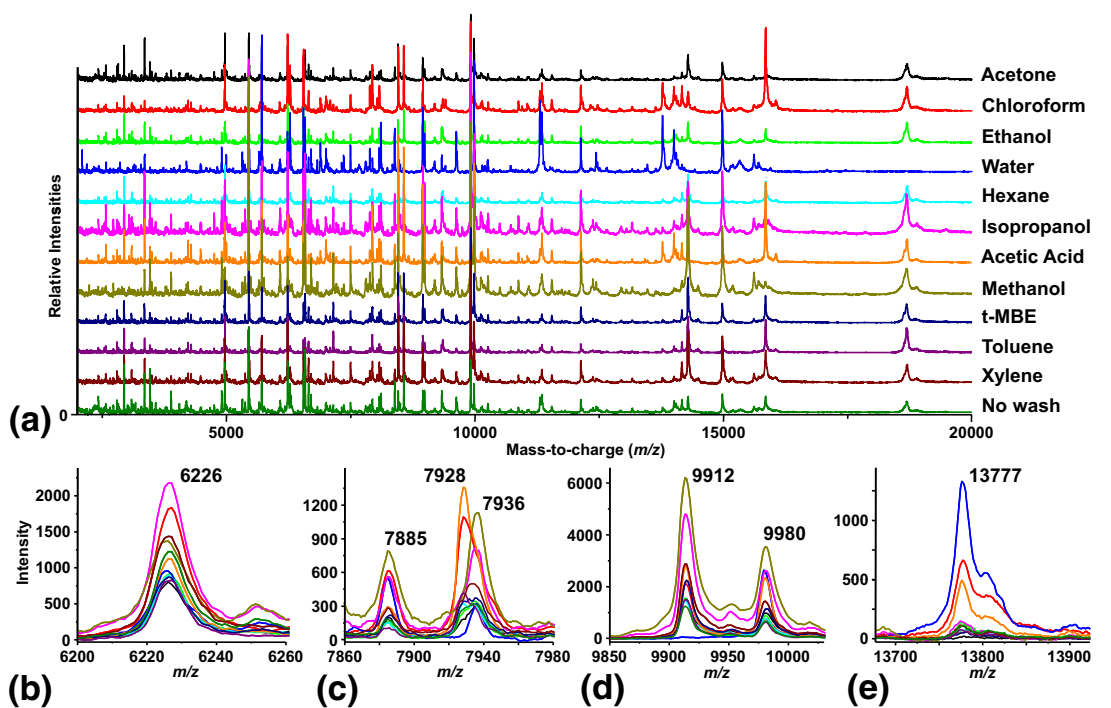

Figure 1. Average MALDI-TOF MS protein profiles directly acquired from serial mouse liver tissue sections not washed or washed with different solvent systems, (a) full mass range, (b-e) selected mass signals showing specific behaviors for the different washes (see text for details).

and analyzed for lipid content in both positive- and negative-ion reflector modes. The resulting average traces for the positive mode analysis are shown in Supplemental Figure S1 (which can be found in the electronic version of this article). All of the alcoholbased washes showed spectra practically devoid of discernable peaks and had the lowest calculated TIC. A similar trend was observed for the negative-mode lipid analysis (not shown). Lipid TIC values (integrated between $\mathrm{m} / \mathrm{z} 500$ and 1100) were plotted on the same graph and in the order as previously obtained for protein TIC in Figure 2a. Again a simple linear fit was drawn to guide the eye. Interestingly, the lipid TIC trend is roughly opposite that observed from the protein analysis, indicating that the removal of lipids from the tissue enhances the observation of protein signals. This can be tentatively explained by (1) the disruption of the cell membranes and removal of the lipids allows better access to intracellular proteins [24] and (2) since both lipids and proteins compete for charge, if one species is removed the measured abundance of the other should increase. Taken together, these results lead to the conclusion that the alcohols as well as acetic acid and water washes were the most beneficial to protein mass spectral analysis from liver tissue sections and warranted further investigation.
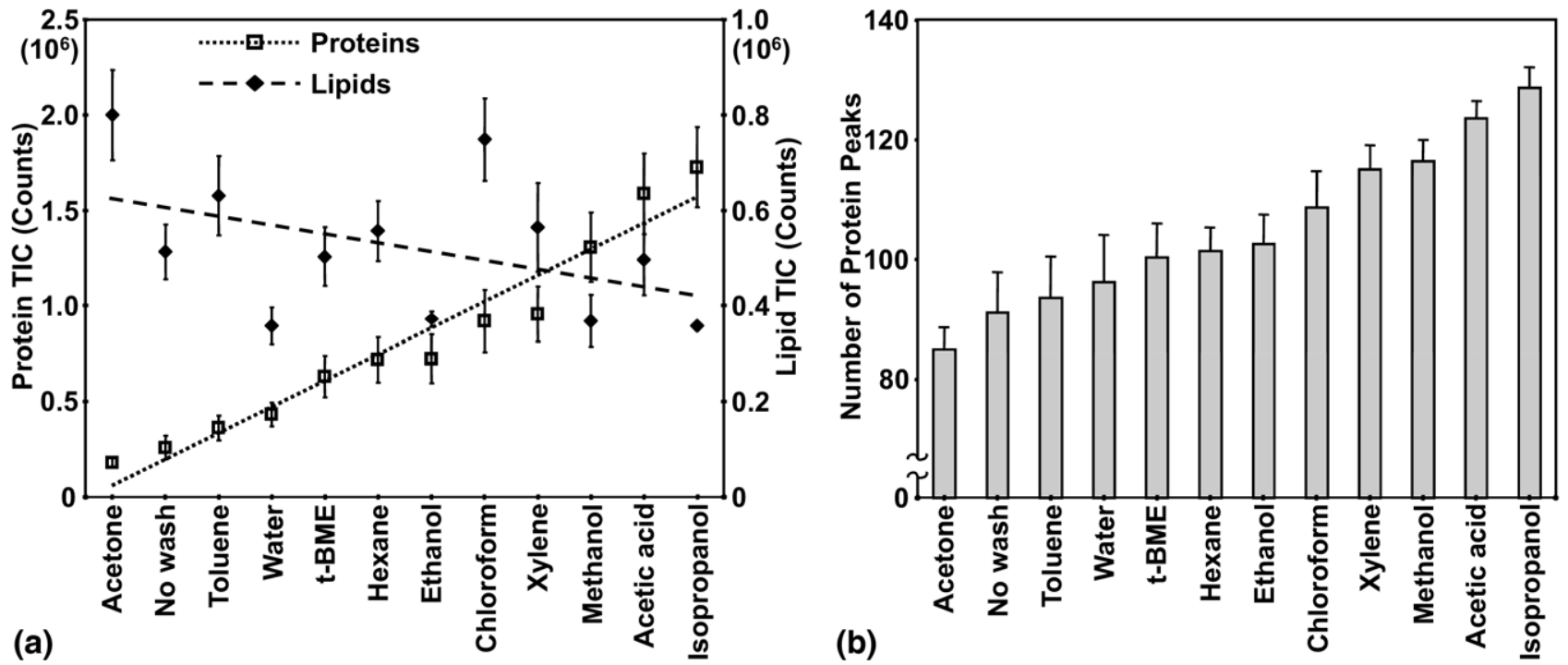

Figure 2. (a) TIC variations recorded from the MALDI-TOF MS protein profiles acquired from serial mouse liver tissue sections not washed or washed with different solvent systems in the $\mathrm{m} / \mathrm{z}$ range from 500 to 1100 (lipid component) and $m / z$ range from 2000 to 25,000 (protein component). (b) Number of peak variations as a function of the same washes for the protein component. 
Four washes (ethanol, isopropanol, acetic acid, and water) were further evaluated for their ability to extend the viable life of tissue sections for direct tissue analysis by MALDI-MS. Serial sections cut from the same mouse liver were washed the day they were sectioned and placed into the desiccator until analysis. As a control, a second set of tissue sections was left unwashed. Tissue sections from each wash were again spotted with 25 matrix spots in a $5 \times 5$ array and mass spectra acquired on each of Day 1 (the day the tissue was sectioned), Day 2, Day 4, Day 7, and Day 14 postsectioning. Spectra were baseline corrected, aligned, and averaged per day and wash type. Again, the TIC was evaluated and plotted as a function of time for each of the washes as seen in Supplemental Figure S2. To emphasize variations with time, relative TIC yields were plotted with respect to Day 1. Only the unwashed tissue showed a drop off in signal intensity of about $65 \%$ on Day 4 and later in contrast to all of the washed tissues that showed no signs of TIC decrease with respect to Day 1 over the time course of the study. Number of peaks was also considered in the analysis, but proved not to be an accurate representation of the process because tissue degradation with time resulted in an increase in the number of peaks observed in the low-mass region $(\mathrm{m} / \mathrm{z}<$ 4000), thus biasing these results (see following text). Washing with isopropanol appeared to give the best tissue preservation with the Day 7 spectrum practically indistinguishable from the Day 1 spectrum, as shown in Figure 3a. The Day 14 isopropanol spectrum showed signs of decreased signal resolution across the studied mass range. When looking in detail at the protein signal from the unwashed tissue, these rapidly deteriorated over time (Supplemental Figure S2 and Figure 3b). Most of the signals showed a decrease in intensity as a function of time, whereas some completely disappeared. For example, the protein signal detected at $m / z$ 12,131 (most likely cytochome c) is clearly observed only up to Day 4. Another example is shown in the insets beside Figure 3a and $b$ showing the same set of signals centered around $m / z 6600$ (arrows in Figure 3a and b), which remained fairly constant in the isopropanol-washed tissue but decreased dramatically in the unwashed tissue. The unwashed tissue also showed an increase in the signals observed in the low-mass region of the spectrum $(\mathrm{m} / \mathrm{z}<$ 4000) likely as the result of protein degradation. It is concluded that the lifetime of the sections can be significantly extended, up to 14 days, after washing with the four chosen solvent systems.

These same four washes were assessed for their ability to "revive" tissue that had been allowed to age in a desiccator after sectioning. In this case, the tissue was sectioned on Day 1 and was not washed until the day it was spotted and analyzed (Day 1, 2, 4, 7, or 14). Example spectra are shown in Supplemental Figure S3 for Days 2 and 7. Although the Day 2 spectra are fairly comparable to the unwashed control, the Day 7 revived tissue showed considerable degradation, especially resulting in an increase in the detection of low-mass signals. Figure 4 shows a selected set of peaks from the isopropanol-washed and the water-washed tissue over the full time course of the experiment. A clear loss of both signal intensity and resolution started to be observed after Day 2. From a practical standpoint, it is

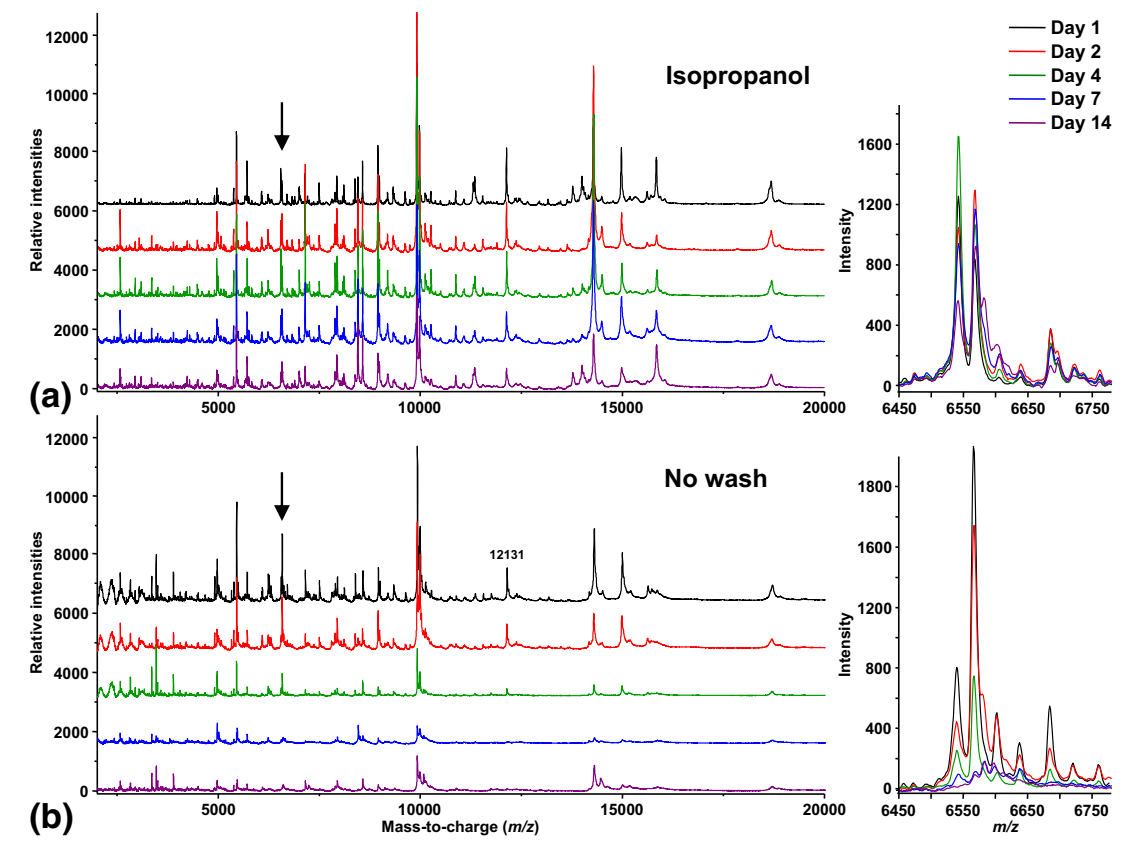

Figure 3. Average MALDI-TOF MS protein profiles acquired from serial mouse liver tissue sections, (a) washed with isopropanol or (b) not washed on Day 1 and spotted with matrix and analyzed on subsequent days. The insets present detailed peak behaviors in the $\mathrm{m} / \mathrm{z}$ range from 6450 to 6750 for each set of sections. 


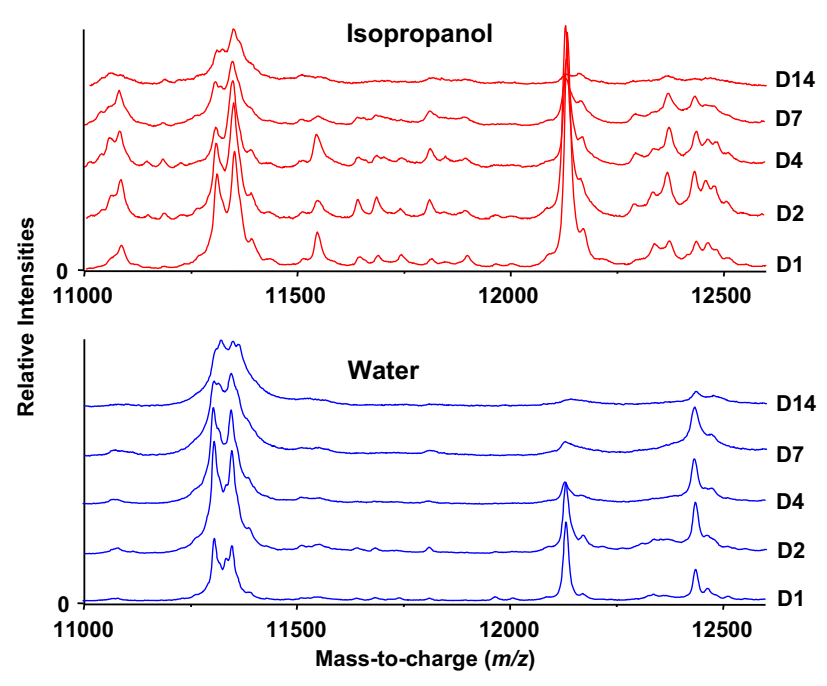

Figure 4. Evolution of average MALDI-TOF MS protein profiles for a selected mass range as a function of time (days) for liver tissue sections cut on Day 1 but washed with isopropanol or water and analyzed on subsequent days.

concluded that tissue sections can be confidently revived by washing one day after sectioning (Day 2) and successfully analyzed without any major noticeable sample alterations.

Additionally, a third experiment was undertaken in which the tissue was not washed, but was spotted the day it was sectioned and then placed in a desiccator for analysis on a later day. The hypothesis behind this is that the tissue will be locally washed by the matrix solution and proteins extracted from the tissue and embedded into the matrix crystals will remain stable. However, as is seen in Supplemental Figure S4, the effects of aging cannot be overcome. A decrease in overall intensity is observed over time (inset) and some signals disappear completely as the matrix spots aged. It is therefore concluded that for maximum ion yield recovery and optimal spectrum quality, MALDI-MS analyses of unwashed tissues should be performed as soon as possible after matrix deposition.

For each wash, in addition to the intensity and number of signals observed, it is important to consider matrix deposition behavior and histological integrity of the tissue for sections that may be destined to be stained post MS analyses. This was monitored by examining the regularity of matrix spot arrays, the crystal formation within a spot, and the histology on a stained tissue section. Crystal architecture was explored by examining the matrix spots deposited on tissue. Figure 5a shows photomicrographs of $5 \times 5$ grids of printed matrix spots on tissues previously subjected to each of the four selected washing procedures and the control unwashed tissue. Unwashed, isopropanol, and acetic acid showed very regular arrays with no spots merging. Ethanol showed a somewhat irregular array where several spots have merged. For water, the array observed was clearly misaligned with multiple spots merging because of delocalization and larger spot size. When examining the crystals from single spots (Figure 5b), unwashed, ethanol, and isopropanol looked very similar with long spindly crystals. Crystal coverage on the acetic acidwashed tissue was sparser and the water-washed tissue showed smaller, more round crystals.

To examine the histology, serial liver sections were collected on glass microscope slides and subjected to the four selected tissue washes before standard H\&E staining. As a control, a fifth section was stained without prior washing. The stained sections were then evaluated for the effects of the washes. Representative pictures demonstrating the histology are shown at an equivalent $\times 4$ (Figure 5c) and $\times 40$ (Figure $5 \mathrm{~d}$ ) magnification. Ethanol and isopropanol were found to be nearly indistinguishable from the unwashed control. Cell membranes, nuclei, as well as nucleoli could clearly be distinguished within the sections. The acetic acid wash had some disruptive effect on the tissue. Although membranes and nuclei could still be observed, the cells appeared blurred and the nuclei did not stain as intensely as those in the other alcohol-based washes. Histological analysis on this tissue section would be compromised as compared to control. The water wash completely destroyed the histology of the tissue section. Holes were observed within the section and cells could no longer be distinguished, nor could any other features present within the tissue such as the duct visible in the upper left D panel of the other washes. It is concluded that the alcohols (ethanol and isopropanol) are the most conducive to histological staining of fresh-frozen tissue sections.

Finally, an imaging experiment was carried out to visualize the effects of washing on the tissue with regard to signals observed as well as delocalization of proteins (Figure 6). For this experiment, a human clear cell renal cell carcinoma section was used because it showed different histological regions as opposed to liver tissue, which is very homogeneous. The collected

(a)
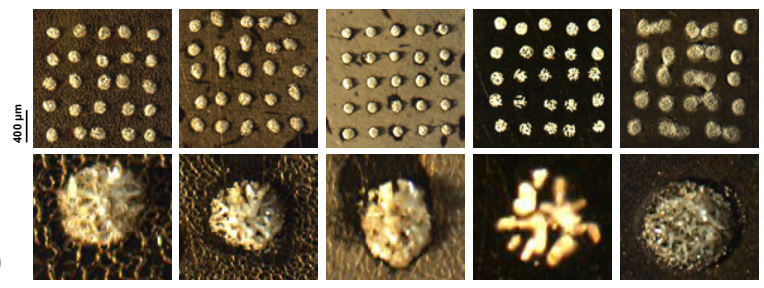

(c)
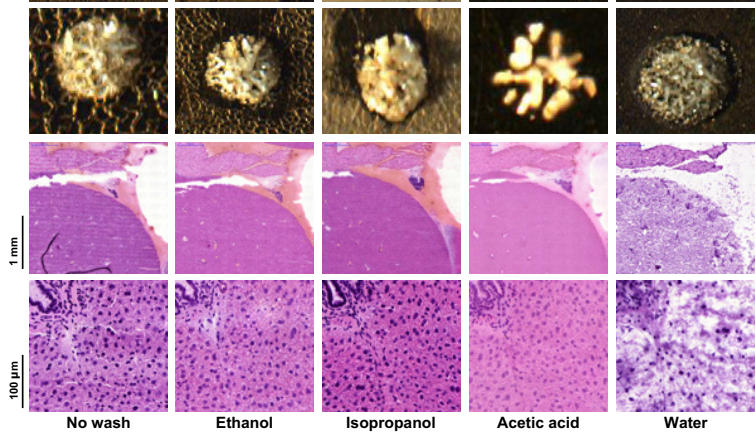

(d)

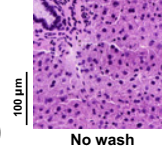

Ethanol

Isopropano

Acetic acid

Water

Figure 5. Magnified photomicrographs of liver tissue sections nonwashed and washed with isopropanol, ethanol, acetic acid, or water after matrix spot array automated deposition (a, b), and H\&E staining (c, d). See text for details. 
(a)

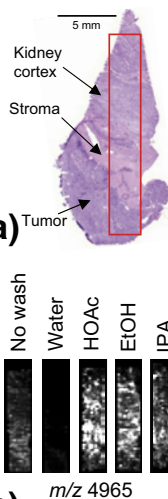

(e) $\begin{gathered}m / z 4965 \\ \text { Thymosin } \\ \square\end{gathered}$
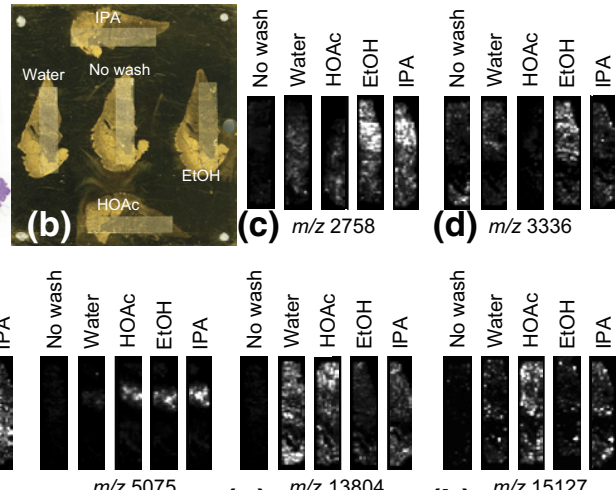

(f) $m / z 5075$ (C) $m / z 2758$
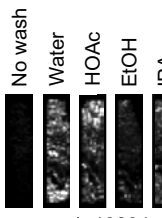

$\mathrm{m} / \mathrm{z} 13804$

(g) Histone H2B1 (d) $m / 23336$

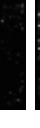

(h) $\mathrm{m} / \mathrm{z} 15127$

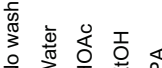

Figure 6. IMS analyses of serial sections cut from a clear cell renal cell carcinoma resection not washed or after washing with isopropanol, ethanol, acetic acid, or water. (a) Photomicrograph of a serial H\&E stained section detailing the histology of the biopsy. Areas of normal kidney cortex, stroma, and tumor are visible. The area boxed in red was analyzed. (b) Photomicrograph of the sections used for IMS after washing and automated matrix deposition. The sections on the edge of the target were washed, whereas the center section was left unwashed. (c-h) Ion density maps for selected protein signals from the nonwashed and washed sections. HOAc, acetic acid; EtOH, ethanol; IPA, isopropanol.

sections contained areas of tumor, stroma, normal kidney cortex, and a highly vascularized region within the cortex (Figure 6a). Five serial sections of the tissue block were collected on one MALDI target plate with one section at each of the edges and one in the center (Figure $6 b)$. By submerging only part of the plate in the wash solutions, each of the sections at the edge of the plate was subjected to one of each of the ethanol, isopropanol, acetic acid, and water washes. The tissue section at the center of the plate served as the unwashed control. Each of the sections was spotted over approximately the same area (Figure 6a) with a $55 \times 12$ spot array with a lateral spacing of $300 \mu \mathrm{m}$ that transversed areas of tumor, stroma, cortex, and vascularization. After IMS data acquisition, as was the case in the profiling experiments, a number of differences in the observed proteins were seen in the resulting images. Figure $6 c-h$ summarizes the IMS results from this experiment. Of note, the protein signals detected at $\mathrm{m} / \mathrm{z} 2758,5075$, and 13,804 were not observed in the unwashed tissue; these appear only after washing. For all of the monitored signals, the alcohol-based washes (ethanol and isopropanol) showed very similar protein distributions and intensities. Several ion density images were clearly affected by some of the washes. Washing with acetic acid resulted in the decrease or loss of some signals such as those detected at $\mathrm{m} / \mathrm{z} 2758$ and 3336. As observed in the profiling studies, the water wash had the greatest impact on the proteins observed and resulted in the complete loss of some signals such as that detected at $\mathrm{m} / \mathrm{z} 4965$ (likely to correspond to thymosin $\beta 4)$, one of the most intense signals observed in the sections subjected to the other washes. Although one of the aims with tissue washing is the removal of hemo-

globin from residual blood in the sample, the images obtained for the alpha chain $(m / z ~ 15,127)$ indicate that this is not the case. In fact, all of the washes showed enhanced hemoglobin signal compared with that of the unwashed tissue. Based on the sum of results from the IMS experiment, protein delocalization across the sections did not appear to be a problem with any of the studied washes. It is believed that considering the volume of solvent used and the fact that the sections were fully submerged, proteins soluble in the wash solutions were completely removed from the surface of the tissue and did not redeposit at another location on the section or the target plate in any significant amounts.

\section{Conclusions}

The results presented here represent an important first step in the optimization of tissue-washing protocols before direct MALDI-MS analysis of proteins. In this study, we found that alcohol-based washes of sections, in particular isopropanol, to be most effective for protein analysis when considering MS signal quality, matrix deposition regularity, and preservation and histological integrity of the tissue. These solvents were also found to effectively remove lipids from the tissue, the molecules most likely to significantly compete with proteins for ionization. A strong correlation was found between the effectiveness of the wash in removing lipids from the section and the detection of optimal protein signals. The fact that alcohol-based washes allow preservation of the sections for protein analysis for several days is very important if it becomes necessary to ship samples to another institution. This event may happen when working with a pathologist offsite for histology-directed analysis, where it may take several days to annotate sections for areas to analyze. However, there are still many avenues that need to be explored in the realm of tissue washing. A detailed analysis was carried out here for liver tissue. Other than for the alcohol- and acid-based washes, which contained some percentage of water, other solvent combinations were not explored. Although alcohol-based washing procedures tested for liver were also found appropriate for the study of a clear cell renal cell carcinoma resection, possibly other solvents or mixtures of solvents may be found optimal for other organs. Optimization of tissue preparation is an ongoing process with the ultimate goal being high-quality, reproducible mass spectral data.

\section{Acknowledgments}

The authors gratefully acknowledge Kristina Schwamborn, M.D. (Universitätsklinikum der RWTH Aachen, Germany) for her help with the histological evaluation of the H\&E stained sections. The authors also acknowledge funding from the National Cancer Institute (Grant 5R33 CA-116123-3) and the National Institutes of Health (Grant 5R01 GM-058008-09). 


\section{References}

1. Stoeckli, M.; Chaurand, P.; Hallahan, D. E.; Caprioli, R. M. Imaging Mass Spectrometry: A New Technology for the Analysis of Protein Expression in Mammalian Tissues. Nat. Med. 2001, 7, 493-496.

2. Chaurand, P.; Schwartz, S. A.; Caprioli, R. M. Profiling and Imaging Proteins in Tissue Sections by Mass Spectrometry. Anal. Chem. 2004, 76, 86A-93A

3. Chaurand, P.; Norris, J. L.; Cornett, D. S.; Mobley, J. A.; Caprioli, R. M. New Developments in Profiling and Imaging of Proteins from Tissue Sections by MALDI Mass Spectrometry. J. Proteome Res. 2006, 5, 28892900.

4. Cornett, D. S.; Mobley, J. A.; Dias, E. C.; Andersson, M.; Arteaga, C. L.; Sanders, M. E.; Caprioli, R. M. A Novel Histology-directed Strategy for MALDI-MS Tissue Profiling That Improves Throughput and Cellular Specificity in Human Breast Cancer. Mol. Cell. Proteomics 2006, 5, 1975-1983

5. Jackson, S. N.; Wang, H. Y. J.; Woods, A. S. Direct Profiling of Lipid Distribution in Brain Tissue Using MALDI-TOFMS. Anal. Chem. 2005, 77, 4523-4527

6. Garrett, T. J.; Prieto-Conaway, M. C.; Kovtoun, V.; Bui, H.; Izgarian, N.; Stafford, G.; Yost, R. A. Imaging of Small Molecules in Tissue Sections with a New Intermediate-Pressure MALDI Linear Ion Trap Mass Spectrometer. Int. J. Mass Spectrom. 2007, 260, 166-176.

7. Verhaert, P.; Uttenweiler-Joseph, S.; de Vries, M.; Loboda, A.; Ens, W.; Standing, K. G., Matrix-Assisted Laser Desorption/Ionization Quadrupole Time-of-Flight Mass Spectrometry: An Elegant Tool for Peptidomics. Proteomics 2001, 1, 118-131.

8. Fournier, I.; Day, R.; Salzet, M. Direct Analysis of Neuropeptides by In Situ MALDI-TOF Mass Spectrometry in the Rat Brain. Neuroendocrinol. Lett. 2003, 24, 9-14.

9. Kruse, R.; Sweedler, J. V. Spatial Profiling Invertebrate Ganglia Using MALDI MS. J. Am. Soc. Mass Spectrom. 2003, 14, 752-759.

10. DeKeyser, S. S.; Kutz-Naber, K. K.; Schmidt, J. J.; Barrett-Wilt, G. A.; Li, L. J. Imaging Mass Spectrometry of Neuropeptides in Decapod Crustacean Neuronal Tissues. J. Proteome Res. 2007, 6, 1782-1791.

11. Chaurand, P.; Schwartz, S. A.; Caprioli, R. M. Imaging Mass Spectrometry: A New tool to Investigate the Spatial Organization of Peptides and Proteins in Mammalian Tissue Sections. Curr. Opin. Chem. Biol. 2002, 6, 676-681.

12. Reyzer, M. L.; Hsieh, Y.; Ng, K.; Korfmacher, W. A.; Caprioli, R. M. Direct Analysis of Drug Candidates in Tissue by Matrix-Assisted Laser Desorption/Ionization Mass Spectrometry. J. Mass Spectrom. 2003, 38, 1081-1092.

13. Reyzer, M. L.; Caldwell, R. L.; Dugger, T. C.; Forbes, J. T.; Ritter, C. A.; Guix, M.; Arteaga, C. L.; Caprioli, R. M. Early Changes in Protein Expression Detected by Mass Spectrometry Predict Tumor Response to Molecular Therapeutics. Cancer Res. 2004, 64, 9093-9100.

14. Khatib-Shahidi, S.; Andersson, M.; Herman, J. L.; Gillespie, T. A.; Caprioli, R. M. Direct Molecular Analysis of Whole-Body Animal Tissue Sections by Imaging MALDI Mass Spectrometry. Anal. Chem. 2006, 78, 6448-6456.

15. Stoeckli, M.; Staab, D.; Schweitzer, A. Compound and Metabolite Distribution Measured by MALDI Mass Spectrometric Imaging in Whole-Body Tissue Sections. Int. J. Mass Spectrom. 2007, 260, 195-202.

16. Chaurand, P.; Caprioli, R. M. Direct Profiling and Imaging of Peptides and Proteins from Mammalian Cells and Tissue Sections by Mass Spectrometry. Electrophoresis 2002, 23, 3125-3135.

17. Cornett, D. S.; Reyzer, M. L.; Chaurand, P.; Caprioli, R. M. MALDI Imaging Mass Spectrometry: Molecular Snapshots of Biochemical Systems. Nat. Methods 2007, 4, 828-833.

18. Chaurand, P.; Sanders, M. E.; Jensen, R. A.; Caprioli, R. M. Proteomics in Diagnostic Pathology: Profiling and Imaging Proteins Directly in Tissue Sections. Am. J. Pathol. 2004, 165, 1057-1068.

19. Chaurand, P.; Schwartz, S. A.; Caprioli, R. M. Assessing Protein Patterns in Disease Using Imaging Mass Spectrometry. J. Proteome Res. 2004, 3, 245-252.

20. Chaurand, P.; Schwartz, S. A.; Reyzer, M. L.; Caprioli, R. A. Imaging Mass Spectrometry: Principles and Potentials. Toxicol. Pathol. 2005, 33, 92-101.

21. Schwartz, S. A.; Reyzer, M. L.; Caprioli, R. M. Direct Tissue Analysis Using Matrix-Assisted Laser Desorption/Ionization Mass Spectrometry: Practical Aspects of Sample Preparation. J. Mass Spectrom. 2003, 38, 699-708.

22. Sugiura, Y.; Shimma, S.; Setou, M. Thin Sectioning Improves the Peak Intensity and Signal-to-Noise Ratio in Direct Tissue Mass Spectrometry. J. Mass Spectrom. Soc. Jpn. 2006, 54, 45-48.

23. Chaurand, P.; Schwartz, S. A.; Billheimer, D.; Xu, B. J.; Crecelius, A.; Caprioli, R. M. Integrating Histology and Imaging Mass Spectrometry. Anal. Chem. 2004, 76, 1145-1155.

24. Lemaire, R.; Wisztorski, M.; Desmons, A.; Tabet, J. C.; Day, R.; Salzet, M.; Fournier, I. MALDI-MS Direct Tissue Analysis of Proteins: Improving Signal Sensitivity Using Organic treatments. Anal. Chem. 2006, 78, 7145-7153.

25. Stoeckli, M.; Staab, D.; Staufenbiel, M.; Wiederhold, K.-H.; Signor, L. Molecular Imaging of Amyloid Beta Peptides in Mouse Brain Sections Using Mass Spectrometry. Anal. Biochem. 2002, 311, 33-39.
26. Aerni, H. R.; Cornett, D. S.; Caprioli, R. M. Automated Acoustic Matrix Deposition for MALDI Sample Preparation. Anal. Chem. 2006, 78, 827-834.

27. Sugiura, Y.; Shimma, S.; Setou, M. Two-Step Matrix Application Technique to Improve Ionization Efficiency for Matrix-Assisted Laser Desorption/Ionization in Imaging Mass Spectrometry. Anal. Chem. 2006, $78,8227-8235$.

28. Groseclose, M. R.; Andersson, M.; Hardesty, W. M.; Caprioli, R. M. Identification of Proteins Directly from Tissue: In Situ Tryptic Digestions Coupled with Imaging Mass Spectrometry. J. Mass Spectrom. 2007, $42,254-262$.

29. Shimma, S.; Furuta, M.; Ichimura, K.; Yoshida, Y.; Setou, M. Direct MS/MS Analysis in Mammalian Tissue Sections Using MALDI-QITTOFMS and Chemical Inkjet Technology. Surf. Interface Anal. 2006, 38, 1712-1714.

30. Lemaire, R.; Desmons, A.; Tabet, J. C.; Day, R.; Salzet, M.; Fournier, I. Direct Analysis and MALDI Imaging of Formalin-Fixed, ParaffinEmbedded Tissue Sections. J. Proteome Res. 2007, 6, 1295-1305.

31. Spengler, B.; Hubert, M. Scanning Microprobe Matrix-Assisted Laser Desorption Ionization (SMALDI) Mass Spectrometry: Instrumentation for Sub-micrometer Resolved LDI and MALDI Surface Analysis. J. Am. Soc. Mass Spectrom. 2002, 13, 735-748.

32. Chaurand, P.; Schriver, K. E.; Caprioli, R. M. Instrument Design and Characterization for High Resolution MALDI-MS Imaging of Tissue Sections. J. Mass Spectrom. 2007, 42, 476-489.

33. Luxembourg, S. L.; Mize, T. H.; McDonnell, L. A.; Heeren, R. M. A High-Spatial Resolution Mass Spectrometric Imaging of Peptide and Protein Distributions on a Surface. Anal. Chem. 2004, 76, 5339-5344.

34. Holle, A.; Haase, A.; Kayser, M.; Höhndorf, J. Optimizing UV Laser Focus Profiles for Improved MALDI Performance. J. Mass Spectrom. 2006, 41, 705-716.

35. Verhaert, P. D.; Conaway, M. C. P.; Pekar, T. M.; Miller, K. Neuropeptide Imaging on an LTQ with vMALDI Source: The Complete "All-inOne" Peptidome Analysis. Int. J. Mass Spectrom. 2007, 260, 177-184.

36. Taban, I. M.; Altelaar, A. F. M.; Van der Burgt, Y. E. M.; McDonnell, L. A.; Heeren, R. M. A.; Fuchser, J.; Baykut, G. Imaging of Peptides in the Rat Brain Using MALDI-FTICR Mass Spectrometry. J. Am. Soc. Mass Spectrom. 2007, 18, 145-151.

37. Stoeckli, M.; Farmer, T. B.; Caprioli, R. M. Automated Mass Spectrometry Imaging with a Matrix-Assisted Laser Desorption Ionization Timeof-Flight Instrument. J. Am. Soc. Mass Spectrom. 1999, 10, 67-71.

38. McCombie, G.; Staab, D.; Stoeckli, M.; Knochenmuss, R. Spatial and Spectral Correlations in MALDI Mass Spectrometry Images by Clustering and Multivariate Analysis. Anal. Chem. 2005, 77, 6118-6124.

39. Norris, J. L.; Cornett, D. S.; Mobley, J. A.; Andersson, M.; Seeley, E. H.; Chaurand, P.; Caprioli, R. M. Processing MALDI Mass Spectra to Improve Mass Spectral Direct Tissue Analysis. Int. J. Mass Spectrom. 2007, 260, 212-221

40. Klerk, L. A.; Broersen, A.; Fletcher, I. W.; van Liere, R.; Heeren, R. M. A. Extended Data Analysis Strategies for High Resolution Imaging MS New Methods to Deal with Extremely Large Image Hyperspectral Datasets. Int. J. Mass Spectrom. 2007, 260, 222-236.

41. Klinkert, I.; McDonnell, L. A.; Luxembourg, S. L.; Altelaar, A. F. M. Amstalden, E. R.; Piersma, S. R.; Heeren, R. M. A. Tools and Strategies for Visualization of Large Image Data Sets in High-Resolution Imaging Mass Spectrometry. Rev. Sci. Instrum. 2007, 78, 053716-1-053716-10.

42. Bloor, W. R. The Determination of Small Amounts of Lipid in Blood Plasma. J. Biol. Chem. 1928, 77, 53-73.

43. Folch, J.; Lees, M.; Stanley, G. H. S. A Simple Method for the Isolation and Purification of Total Lipides from Animal Tissues. J. Biol. Chem. 1957, 226, 497-509.

44. Bligh, E. G.; Dyer, W. J. A Rapid Method of Total Lipid Extraction and Purification. Can. J. Biochem. Physiol. 1959, 37, 911-917.

45. Iverson, S. J.; Lang, S. L. C.; Cooper, M. H. Comparison of the Bligh and Dyer and Folch Methods for Total Lipid Determination in a Broad Range of Marine Tissue. Lipids 2001, 36, 1283-1287.

46. Jiang, L.; He, L.; Fountoulakis, M. Comparison of Protein Precipitation Methods for Sample Preparation Prior to Proteomic Analysis. J. Chromatogr. A 2004, 1023, 317-320.

47. Christie, W. W. Preparation of Lipid Extracts from Tissues. In Advances in Lipid Methodology-Two, Christie, W. W., Ed.; Oily Press: Dundee, Scotland, 1993; pp 195-213.

48. Hanahan, D. J. A Guide to Phospholipid Chemistry; Oxford University Press: Oxford, UK, 1997.

49. Maneta-Peyret, L.; Compere, P.; Moreau, P.; Goffinet, G.; Cassagne, C. Immunocytochemistry of Lipids: Chemical Fixatives Have Dramatic Effects on the Preservation of Tissue Lipids. Histochem. J. 1999, 31, 541-547.

50. Wessel, D.; Flugge, U. I. A Method for the Quantitative Recovery of Protein in Dilute Solution in the Presence of Detergents and Lipids. Anal. Biochem. 1984, 138, 141-143.

51. Prophet, E. B.; Mills, B.; Arrington, J. B.; Sobin, L. H., Eds. Laboratory Methods in Histotechnology. American Registry of Pathology: Washington, D.C., 1994

52. EmmertBuck, M. R.; Bonner, R. F.; Smith, P. D.; Chuaqui, R. F.; Zhuang, Z. P.; Goldstein, S. R.; Weiss, R. A.; Liotta, L. A. Laser Capture Microdissection. Science 1996, 274, 998-1001. 